\title{
Estimation of musculotendon kinematics in large musculoskeletal models using multidimensional B-Splines
}

\author{
Massimo Sartoria,b, ${ }^{,}$, Monica Reggianic $^{c}$, Antonie J. van den Bogert ${ }^{d}$, and David G. Lloyd ${ }^{\mathrm{e}}$ \\ ${ }^{a}$ Dept of Information Engineering, University of Padova, Via Gradenigo 6/B, Padova, IT 35131, \\ Italy \\ bInstitute of Biomedical Engineering, National Research Council, Corso Stati Uniti 4, Padova, IT \\ 35127, Italy \\ 'Dept of Management and Engineering, University of Padova, Stradella S. Nicola 3, Vicenza, IT \\ 36100 , Italy \\ 'Orchard Kinetics LLC, Cleveland, OH, USA \\ eMusculoskeletal Research Program, Griffith Health Institute, Griffith University, Gold Coast, \\ QLD, 4222, Australia
}

\begin{abstract}
We present a robust and computationally inexpensive method to estimate the lengths and threedimensional moment arms for a large number of musculotendon actuators of the human lower limb. Using a musculoskeletal model of the lower extremity, a set of values was established for the length of each musculotendon actuator for different lower limb generalized coordinates (joint angles). A multidimensional spline function was then used to fit these data. Muscle moment arms were obtained by differentiating the musculotendon length spline function with respect to the generalized coordinate of interest. This new method was then compared to a previously used polynomial regression method. Compared to the polynomial regression method, the multidimensional spline method produced lower errors for estimating musculotendon lengths and moment arms throughout the whole generalized coordinate workspace. The fitting accuracy was also less affected by the number of dependent degrees of freedom and by the amount of experimental data available. The spline method only requires information on musculotendon lengths to estimate both musculotendon lengths and moment arms, thus relaxing data input requirements, whereas the polynomial regression requires different equations to be used for both musculotendon lengths and moment arms. Finally, we used the spline method in conjunction with an electromyography driven musculoskeletal model to estimate muscle forces under different contractile conditions, which showed the method is suitable for the integration into large scale neuromusculoskeletal models.
\end{abstract}

\footnotetext{
(c) 2011 Elsevier Ltd. All rights reserved.

*Corresponding Author University of Padova, Department of Information Engineering, Intelligent Autonomous Systems Laboratory (Stanza L3), Via Ognissanti 72, Padova, IT 35131 Italy, Phone: +39 04982778 33, Mobile: +39 34961564 10, Fax: +39 04982778 26, massimo.srt@gmail.com, URL: http://www.massimosartori.net/.

Conflict of interest statement There were not conflicts of interest between these funding bodies and procedures and results in this study.

Publisher's Disclaimer: This is a PDF file of an unedited manuscript that has been accepted for publication. As a service to our customers we are providing this early version of the manuscript. The manuscript will undergo copyediting, typesetting, and review of the resulting proof before it is published in its final citable form. Please note that during the production process errors may be discovered which could affect the content, and all legal disclaimers that apply to the journal pertain.
} 


\section{Keywords}

musculoskeletal modeling; musculotendon length; muscle moment arm; muscle force; multidimensional spline interpolation

\section{Introduction}

Musculoskeletal modeling has applications ranging from the study of muscle contributions to movement (Lloyd and Besier, 2003; Winby et al., 2009) to the development of humanmachine interfaces (Fleischer and Hommel, 2008). Musculoskeletal modeling and simulations require accurate estimates of musculotendon kinematics including musculotendon length $\left(\ell^{m t}\right)$ and three-dimensional moment arms $(r)$ to accurately predict musculotendon forces $\left(F^{m t}\right)$ and joint moments.

Musculotendon kinematics estimates can be produced by software that models musculotendon paths wrapping around points and/or surfaces (Delp et al., 2007; Delp et al., 1990). However, this is based on obstacle detection and may cause discontinuities in the predicted musculotendon kinematics (Gao et al., 2002; Garner and Pandy, 2000). Moreover, it is desirable that musculotendon kinematics equations are continuously differentiable to enable the computation of analytical Jacobians for the forward simulation of the musculoskeletal system (Ackermann and van den Bogert, 2010; Menegaldo et al., 2006).

Sets of differentiable polynomial regression equations $\left(P^{e q}\right)$ have been proposed to estimate $\ell^{m t}$ and $r$ from nominal values of both parameters corresponding to combinations of discrete joint angles or generalized coordinates (GC) (Menegaldo et al., 2004). This required gathering data sets on both $\ell^{m t}$ and $r$ and manually identifying the best-performing equations that depended on the muscle, number of GCs, and the fitted musculotendon parameter. This necessitated computing and storing coefficients for all equations for $\ell^{m t}$ and $r$ for each muscle before use.

Alternatively, continuously differentiable multidimensional cubic splines can be used, but are yet to be examined as a means to estimate musculotendon kinematics. In this paper we show a single spline function per muscle can be used to estimate $\ell^{m t}$ and $r$ based on only nominal values of $\ell^{m t}$. We then compare fitting accuracy and computation time to $P^{e q}$. The $\ell^{m t}$ nominal values were derived from musculoskeletal geometry models using OpenSim (Delp et al., 2007). Finally, we quantified how differences in the $\ell^{m t}$ estimates affect $F^{m t}$ estimates using an electromyography (EMG) driven musculoskeletal model (Buchanan et al., 2004; Lloyd and Besier, 2003).

\section{Methods}

The performance of $P^{e q}$ and spline methods were examined using two musculoskeletal models (Table 1): LowerLimb4307 (Delp et al., 1990) and LowerLimb1310 (Winby et al., 2009). The reader is referred to (Menegaldo et al., 2004) for $P^{e q}$ theory, while a summary of spline theory (Habermann and Kindermann, 2007) is now presented.

A cubic spline consists of a series of third-order polynomials spliced together to preserve continuity of the first and second-order derivatives. The points at which polynomials are spliced together, $a=x_{0}<x_{1}<\ldots<x_{n}=b \in \mathbb{R}$ are called nodes, where $\Delta_{n}=\left[a=x_{0}, x_{1}, \ldots, x_{n}\right.$ $=b]$ is the bounded interval of interest. If $\Delta_{n}$ and the interpolation data $y_{i} \in \mathbb{R}, i=0, \ldots, n$ are given, a spline interpolates $y_{i}$ while satisfying $n+3$ conditions on the nodes: $s_{1}\left(x_{i}\right)=y_{i}$ for $i$ $=0, \ldots, n$, and $\ddot{s}_{1}(a)=\alpha, \ddot{s}_{1}(b)=\beta$, where $s_{1}$ represents a one-dimensional spline function on 
$\Delta_{n}, \ddot{s}_{1}$ is its second-order derivative, while $\alpha$ and $\beta$ are real-valued terms (Habermann and Kindermann, 2007). A spline with $n+1$ nodes can be written as a linear combination of $n+$ 3 cubic polynomials $u_{1}, u_{2}, \ldots, u_{n+3}$ defining a basis:

$$
s_{1}(x)=\sum_{i=l}^{m} c_{i} u_{i}(x)
$$

with coefficients $c_{i}$, where $m=\min (l+3, n+3), l=\left[\frac{x-a}{h}\right]+1$, and $h$ is the distance between nodes. When the interpolation data vary as a function of $d$ variables ( $d$-dimensional case), Eq. (1) can be generalized (Habermann and Kindermann, 2007) thus requiring

$N_{c}=\prod_{i=1}^{d}\left(n_{1}+3\right)$ coefficients whilst a spline value can be computed using

$\prod_{i=1}^{d}\left(m_{i}-l_{i}+1\right) \leq 3^{d}$ coefficients and basis functions.

Given two real-valued variables $x$ and $z$ on the bounded intervals $\Delta_{n_{1}}=\left[a_{1}, b_{1}\right]$ and $\Delta_{n_{2}}=$ $\left[a_{2}, b_{2}\right]$, the spline partial derivative with respect to $x$ can be computed using the spline coefficients $c_{i_{1} i_{2}}$ :

$$
\frac{\partial s_{2}(x, z)}{\partial x}=\sum_{i_{1}=l_{1}}^{m_{1}} \sum_{i_{2}=l_{2}}^{m_{2}} c_{i_{1} i_{2}} \dot{u}_{i_{1}}(x) v_{i_{2}}(z)
$$

where $u_{i_{1}}(x)$ and $v_{i_{2}}(z)$ represent basis functions for the two variables respectively and $u_{i_{1}}$ $(x)$ is the first-order derivative.

The problem of finding $\ell^{m t}$ as a function of $d$ GC-angles, $\left(q_{1}, \ldots, q_{d}\right)$ is formulated by determining the $\left(n_{1}+3\right) \cdot \ldots \cdot\left(n_{d}+3\right)$ coefficients for the $d$-dimensional spline function $s_{d}\left(q_{1}, \ldots, q_{d}\right)$, where $\ell^{m t}$ are the nominal data to be interpolated. Moment arms $\left(r_{q_{i}}\right)$ with respect to a specific GC $\left(q_{i}\right)$ are then found by the partial differentiation (Eq. (2)) of $s_{d}\left(q_{1}\right.$, $\left.\ldots, q_{d}\right)$ :

$$
r_{q_{i}}=\frac{\partial \ell^{m t}\left(q_{1}, \ldots, q_{d}\right)}{\partial q_{i}}=\frac{\partial s_{d}\left(q_{1}, \ldots, q_{d}\right)}{\partial q_{i}}
$$

The $\ell^{m t}$ values for $q_{i}$ that extend beyond their intervals $\Delta_{i}, \forall 1 \leq i \leq d$, are obtained by creating a tangent line at all end points using linear extrapolation with the slope given by the values of $r_{q_{i}}$ at the end points. Moment arms are therefore kept constant for $q_{i}$ values that extend beyond $\Delta_{i}$.

\subsection{Validation procedure}

Validation comprised of four tests to assess fitting accuracy and one to examine computation time and memory storage requirements. GC ranges of motion (ROM) were sampled into equidistant nodes and input into OpenSim ${ }^{1}$ to compute $\ell^{m t}$ and $r$ nominal values for $P^{e q}$ coefficient calculation. Only $\ell^{m t}$ values were used for splines coefficients. The fitting accuracy was assessed both on the interpolation nodes (on-nodes) and on points located midway between nodes (off-nodes).

\footnotetext{
${ }^{1}$ We used the OpenSim 2.2.0 available at: https://simtk.org/home/opensim
} 
The four fitting accuracy tests examined the ability of the spline and $P^{e q}$ methods in reproducing OpenSim-generated $\ell^{m t}, r$ and $F^{m t}$ calculated using OpenSim-generated $\ell^{m t}$ (Section 2.2). The Mean Fitting Error (MFE) and its standard deviation $(\sigma)$ were used to measure the expected error, and its variation, for $P^{e q}$ and spline estimations of OpenSimgenerated values:

$$
\mathrm{MFE}=\frac{1}{N} \sum_{i=1}^{N}\left|\widehat{X}_{i}-X_{i}\right|
$$

where $\hat{X}$ is the OpenSim-generated $\ell^{m t}$ or $r$, or $F^{m t}$ calculated using OpenSim-generated $\ell^{m t}$; the $X$ corresponds to the value estimated using the $P^{e q}$ or spline methods, and $N$ the number of fitting function points. The percent MFE $(\% \varepsilon)$ was estimated with respect to the corresponding expected OpenSim-generated value $(E[\hat{X}])$ :

$$
\% \varepsilon=\frac{\mathrm{MFE}}{E[\widehat{X}]} \times 100
$$

The MFE with $95 \%$ confidence interval (CI) was estimated using the Central Limit Theorem, which enabled maximum expected errors (MEE) to be calculated:

$$
\mathrm{MEE}=\mathrm{MFE}+1.96 \sigma,(95 \%)
$$

This assumed a random error with normal distribution that is generally not the case (Fig. 1a). A normality check was performed using a one-sample Kolmogorov-Smirnov test with a 5\% significance level (Miller, 1956). If normality was rejected, then a more conservative MEE with $90 \%$ and $95 \%$ CIs was obtained using the Chebyshev's Theorem, which can be applied to any probability distribution with known mean and $\sigma$ :

$$
\mathrm{MEE}=\mathrm{MFE}+3.16 \sigma,(90 \%)
$$

$\mathrm{MEE}=\mathrm{MFE}+4.47 \sigma,(95 \%)$

All metrics were applied to all fitting accuracy tests, except for $\% \varepsilon$ that was only used in the second test. These tests are now described in more detail.

\subsection{Validation Fitting Accuracy Tests}

The first test of fitting accuracy examined splines and $P^{e q}$ musculotendon kinematics using Menegaldo's et al. input data, musculoskeletal model (i.e. LowerLimb4307 model), analysis procedure, and resulting $P^{e q}$ set (Menegaldo et al., 2004). GC ROMs were sampled with 20 points, although when $\ell^{m t}$ depended on four GCs, a 15-point sampling was used.

In the second test the semimembranosus was chosen to examine how fitting accuracy changed with a large number of GCs, and varying distance between nodes. The LowerLimb1310 model was used with a 10-point GC ROM sampling using two different ROM arrangements (Table 1). The number of GCs was varied from four to six. The 4-GC set included: hip internal-external rotation (HR), hip adduction-abduction (HA), hip flexionextension (HF), and knee flexion-extension (KF). The 5-GC set added knee internal-external rotation (KR), and the 6-GC set included knee adduction-abduction (KA). 
In the third and fourth tests, biarticular thigh muscles from the LowerLimb1310 model were chosen to assess the impact of the spline and $P^{e q}$ estimates of $\ell^{m t}$ on the calculation of $F^{m t}$. This employed four GCs with knee GCs reduced to KF only, and a 10-point extended ROM sampling (Table 1). $F^{m t}$ was estimated as a function of EMG and $\ell^{m t}$ using an EMG-driven model (Lloyd and Besier, 2003) which we briefly summarize. Raw EMGs were first highpass filtered $(30 \mathrm{~Hz})$, full wave rectified, and low-pass filtered $(6 \mathrm{~Hz})$ to create EMG linear envelopes $(e(t))$. The linear envelopes were normalized to maximum EMG data that were the peak $e(t)$ values from a set of motor tasks that included; three trials of running at $5 \mathrm{~m} / \mathrm{s}$, five repeated calf rises, and maximum exertion isometric knee flexion-extension trials twice repeated at each 100, 70 and 40 degrees of knee flexion on a Biodex (NY, USA) dynamometer. The normalized linear envelopes were then processed using a model that represented the muscle's twitch response as a critically-damped second-order filter (Lloyd and Besier, 2003). These data series $(u(t))$ were subsequently adjusted to account for the non-linear EMG-to-force relationship (Lloyd and Besier, 2003). The resulting muscle activation $(a(t))$ was a dimensionless term varying between 0 and 1 .

Each musculotendon actuator (MTA) had muscle fibers modeled as a Hill-type muscle model (Zajac, 1989) in series with a non-linear elastic tendon (Lloyd and Besier, 2003). Fibers where modeled using generic force-length $f\left(l^{m}\right)$, force-velocity $f\left(v^{m}\right)$, and parallel passive force-length curves $f_{P}\left(l^{m}\right)$. These were normalized to maximum isometric muscle force $\left(F^{\max }\right)$, optimal fiber length $\left(l_{o}^{m}\right)$, and maximum muscle contraction velocity $\left(v^{\max }\right)$ (Zajac, 1989). Tendons were modeled with a non-linear force-strain function $f(\varepsilon)$ (Zajac, 1989), where $\varepsilon=\frac{l^{t}-l_{s}^{t}}{l_{s}^{t}}$ is the tendon strain, $l^{t}$ is the tendon length, and $l_{s}^{t}$ is the tendon slack length, with $f(\varepsilon)$ normalized to $F^{\max }$ (Zajac, 1989). Using biomechanical parameters from (Delp et al., 1990), $F^{m t}$ was calculated as a function of $a(t)$, fiber length $l^{m}$ and fiber contraction velocity $v^{m}$ :

$$
F^{m t}=F^{t}=F^{m} \cos (\phi(t))=\left[a(t) f\left(l^{m}\right) f\left(v^{m}\right)+f_{p}\left(l^{m}\right)\right] F^{\max } \cos (\phi(t)),
$$

where $F^{t}$ is the tendon force, and $\varphi(\mathrm{t})$ is the pennation angle that changes with $l^{m}$ (Scott and Winter, 1991). $\ell^{m t}$ estimates were generated using OpenSim, $P^{e q}$ and splines, which were used as the basis to calculate $F^{m t}$ time series for each MTA (Lloyd and Besier, 2003). Briefly, this was performed using initial values of $l^{m}$ from which tendon length was

determined, i.e. $l^{t}=\ell^{m t}-l^{m} \cos (\varphi(\mathrm{t}))$. Then, tendon strain $\varepsilon=\frac{l^{t}-l_{s}^{t}}{l_{s}^{t}}$ was calculated from which $F^{t}$, and $F^{m t}$, were determined. By rearranging Eq. (9) the fiber velocity dependent muscle force $F^{v}$ was determined (Buchanan et al., 2004):

$$
F^{v}=\frac{\left(F^{m}-f_{P}\left(l^{m}\right) F^{\max }\right)}{a(t) f\left(l^{m}\right) F^{\max }}
$$

since activation $a(\mathrm{t})$ was known and both $f\left(l^{m}\right)$ and $f_{P}\left(l^{m}\right)$ were evaluated using the current $l^{m}$. Using $F^{v}$ and inverting the fiber-velocity relationship $f\left(v^{m}\right), v^{m}$ could be determined, which was then numerically integrated to estimate the next $l^{m}$ in time. And so the process continued until the end of the time series producing estimates for each MTA's time varying $F^{m t}$. In the special case for isometric contractile conditions, a simplified procedure (Buchanan et al., 2004) was used in which initial values for $l^{m}$ and $l^{t}$ were iteratively adjusted to ensure $F^{t}=F^{m} \cos (\varphi(\mathrm{t}))$ and $\ell^{m t}=l^{t}+l^{m} \cos (\varphi(\mathrm{t}))$. 
We compared $F^{m t}$ computed using splines and $P^{e q}$-generated values for $\ell^{m t}$ to our gold standard $F^{m t}$ derived using the OpenSim-generated $\ell^{m t}$. Estimated $F^{m t}$ values were multiplied by the respective muscle KF moment arms and summed to determine the total KF moment.

In the third test the EMG-driven model was used to examine the impact of $\ell^{m t}$ estimates on $F^{m t}$ estimates under maximum isometric contractile conditions (i.e. $a(t)=1$ and $v^{m}(t)=0$, $\forall t$ ) not involving real subject data. On-nodes and off-nodes $\ell^{m t}$ values were calculated using OpenSim, $P^{e q}$, and splines, where each $\ell^{m t}$ value was associated to a hypothetical instant of time $t$.

The fourth test studied how $\ell^{m t}$ estimates affected $F^{m t}$ evaluations in low-effort (walking at $1.4 \pm 0.3 \mathrm{~m} / \mathrm{s}$ ) and higher-effort (jogging at $2.3 \pm 0.4 \mathrm{~m} / \mathrm{s}$ ) dynamic contractile conditions. A healthy male subject (mass: $81.7 \mathrm{Kg}$, height: $175.7 \mathrm{~cm}$, age: 30years) gave his informed, written consent prior to data collection. The subject's static anatomical poses and gait patterns were captured using a seven-camera Vicon MX Motion Analysis system (Oxford Metrics, UK) sampling at $100 \mathrm{~Hz}$. During the gait trials, ground reaction forces (GRF) and EMG data were acquired at $2000 \mathrm{~Hz}$ synchronously with motion data. EMGs were collected from the muscles crossing the knee defined in the LowerLimb1310 model. Marker trajectories and GRF were low-pass filtered $(6 \mathrm{~Hz})$ as suggested by (McLean et al., 2005). OpenSim was used to create a dynamic motion simulation per gait trial. The LowerLimb1310 model was scaled (Delp et al., 2007) to match the subject's anthropometry based on experimentally measured marker positions from static poses. OpenSim created virtual representations of the experimental markers and an inverse kinematics algorithm solved for GC-angles that minimized the difference between experimental and virtual markers (Delp et al., 2007). GC-moments needed to generate the subject's movement were computed using the OpenSim inverse dynamics algorithm (Kuo, 1998). The inverse kinematics GC-angles from the walking and jogging trials were within the bounds of the extended range of motion of the LowerLimb1310 model (Table 1), from which the $\ell^{m t}$ values were computed for each muscle using OpenSim, $P^{e q}$, and splines respectively.

Some EMG-driven model parameters vary non-linearly with subject's anthropometry and the physiological conditions during testing and could not be obtained experimentally or from literature. These parameters were calibrated to the individual (Lloyd and Besier, 2003; Winby et al., 2009) and included two global activation filtering coefficients that were varied between -1 and 1 to realize a stable positive solution to the critically-damped recursive filter (Lloyd and Besier, 2003). One global activation shape parameter was also varied between -5 and 0 to account for the non-linearity of the EMG-to-force relationship (Lloyd and Besier,

2003). Finally, muscle-specific $l_{s}^{t}$ values were varied so that $l_{s}^{t}=$ initial value $\pm 5 \%$, with initial values taken from literature (Delp et al., 1990; Lloyd and Besier, 2003). The parameters were adjusted using a simulated annealing algorithm that minimized $^{2}$ the leastsquared differences between the KF moments estimated using the EMG-driven model and inverse dynamics (Winby et al., 2009). Three walking and two jogging trials were used for parameter adjustment. Using the adjusted parameters the EMG-driven model was then used to estimate $F^{m t}$ (Eq. (9)) in other gait trials not used in the adjustment process. Forces estimated during the stance phase were time-normalized and two ensemble average curves computed from walking and jogging trials respectively and used to estimate the instantaneous force errors using Eq. (4) (with $N=1, \forall 0 \%$ stance $\leq i \leq 100 \%$ stance).

${ }^{2}$ OpenSim-generated $\ell^{m t}$ were used. 
Finally, we examined how computation time varied with the number of GC's. To this end, we computed the average time needed to repeatedly estimate one sample of $\ell^{m t}$ and $r 10000$ times with GCs varying from one to six respectively. A $2.26 \mathrm{GHz} 3 \mathrm{~GB}$-RAM PC was used. Both spline and $P^{e q}$ evaluation routines were implemented in $\mathrm{C}++$ for speed comparison. Furthermore, the memory required to store the coefficients and nominal data for all muscles during each test for both $\mathrm{P}^{e q}$ and spline methods was assessed. For each test and method, two text files were created where coefficients and nominal data were stored respectively, and then the files sizes were reported in megabytes.

\section{Results}

The tibialis anterior muscle was chosen to demonstrate the spline performances in the first test. This muscle's kinematics depended on ankle dorsi-plantar flexion (AF) and subtalar flexion-extension (SF). The variation of $\ell^{m t}$, $r$, and errors are easy to visualize in 3D (Fig. 1). The fitting errors between the OpenSim and spline $\ell^{m t}$ values were uniform throughout the GC workspace reaching a $0.3 \times 10^{-6} \mathrm{~cm}$ maximum while $P^{e q}$ errors reached higher peaks $(0.14 \mathrm{~cm})$ at the boundaries ${ }^{3}$ (Fig. 1a). The $P^{e q}$ fitting error for the AF moment arms reached peak values $(0.59 \mathrm{~cm})$ at the boundaries (Fig. 1b). There was good agreement between the OpenSim-generated surface associated to SF moment arms and those generated by the spline function with a $8 \times 10^{-4} \mathrm{~cm}$ maximum error (Fig. 1c). The distribution of the splinegenerated errors is shown in Figs 1d, 1e and 1f. Errors were in the order of $10^{-5} \mathrm{~cm}$ for the $\ell^{m t}$ estimation and in the order of $10^{-3} \mathrm{~cm}$ for the estimation of AF and SF moment arms.

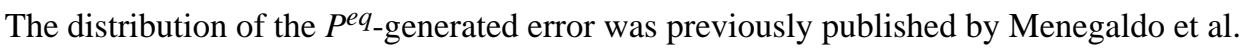
(Menegaldo et al., 2004) and is not reported in this study. However, for all muscles, $P^{e q}$ MFEs were always three orders of magnitude larger than spline MFEs (Table 2).

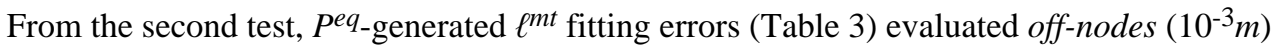
were smaller than those evaluated on-nodes (between $10^{-3} \mathrm{~m}$ and $10^{-2} \mathrm{~m}$ ). On-nodes spline errors were negligible $\left(10^{-10} \mathrm{~m}\right)$, due to the spline interpolation conditions, and off-nodes spline errors varied between $10^{-6} \mathrm{~m}$ and $10^{-4} \mathrm{~m}$ (Table 3). Finally, spline-generated $\% \varepsilon$ for the HA moment arms (Table 4) were smaller (between $0.04 \%$ and $0.26 \%$ ) than $P^{e q}$-generated $\% \varepsilon$ (between $8.38 \%$ and $12 \%$ ).

From the third test, the muscle forces estimated using OpenSim-generated $\ell^{m t}$ are shown in Table 5. $P^{e q}$-generated force errors fell into classes with errors in the hundreds of newtons $(N)$ (Fig. 2a). However, spline-generated force errors were always less than $30 N$ (Fig. 2b) and at all times fell in the first and smallest $P^{e q}$ error class (Fig. 2a).

From the fourth test, the estimated forces generated by semimembranosus in walking and jogging are reported in Table 6. $P^{e q}$-generated force errors were higher during highactivation jogging tasks than during low-activation walking tasks as opposed to the spline case (Fig 2c). The $\ell^{m t}$ fitting errors significantly varied between spline and $P^{e q}$ and directly impacted on the force prediction. During walking, $P^{e q}$-generated $\ell^{m t}$ fitting errors reached peak values $(0.5 \pm 0.07 \mathrm{~cm})$ from $50 \%$ to $70 \%$ of stance corresponding to peak force errors $(4.5 \pm 0.5 N)$ in the same range (Fig. 2c). During jogging, $P^{e q}$-generated $\ell^{m t}$ fitting errors reached peak values $(0.48 \pm 0.02 \mathrm{~cm})$ from $65 \%$ to $80 \%$ of stance corresponding to an increase in force error up to $20 \mathrm{~N}$ in a similar range (Fig 2c). Spline-generated $\ell^{m t}$ fitting errors were always less than $0.006 \mathrm{~cm}$ with corresponding force errors smaller than $1 \mathrm{~N}$ during walking and jogging (Fig. 2c).

\footnotetext{
${ }^{3}$ The polynomial regression equations were from (Menegaldo et al., 2004).
} 
In all tests, the Kolmogorov-Smirnov test rejected the normal distribution hypothesis for splines and $P^{e q}$ fitting errors for all muscles justifying the use of the Chebyshev's Theorem for CI computation.

Spline computation time ranged from $10^{-5} \mathrm{~ms}$ (one-dimensional case) to $10^{-2} \mathrm{~ms}$ (sixdimensional case) as opposed to $10^{-5} \mathrm{~ms}$ with the $P^{e q}$. Even though the number of spline coefficients varies exponentially with the number of GCs and nodes, compared to the $P^{e q}$, the total memory required was smaller in the spline case since splines only needed nominal $\ell^{m t}$ data to estimate both $\ell^{m t}$ and $r$ (Table 7).

\section{Discussion}

Multidimensional cubic B-splines were used to estimate lower-limb musculotendon kinematics and were compared to a previous method that employed polynomial regression (Menegaldo et al., 2004). Splines provided fitting errors that were uniform throughout the GC workspace and at least three orders of magnitude smaller than $P^{e q}$ errors, the latter increasing at the workspace boundaries. $P^{e q}$-generated force errors were i) larger than spline-generated errors during maximum isometric contractile conditions, and ii) dependent on the level of muscle activation during dynamic tasks. This suggests splines may be more reliable for integration into forward dynamics musculoskeletal models, in which analytical Jacobians can be easily computed by differentiating the $\ell^{m t}$ spline function (Eq. (3)).

The time needed to evaluate one point on $P^{e q}$ was always in the order of $10^{-5} \mathrm{~ms}$ whereas spline computation time varied exponentially with the number of GCs. However, this was always less than $0.01 \mathrm{~ms}$ (6-GC case) thus providing adequate speed for applications requiring real-time computation of $\ell^{m t}$ and $r$ with the subsequent real-time estimation of $F^{m t}$ and joint moments. Examples of these emerging applications are biofeedback (Murai et al., 2010) or EMG-controlled powered orthoses (Fleischer and Hommel, 2008) where the realtime deadline is the muscle electromechanical delay of about $20 \mathrm{~ms}$ (Nordez et al., 2009).

In general, spline fitting accuracy increases with the number of nodes with no over-fitting risk. We showed, in tests 1 and 2, that sampling GC-angles with 10, 15 or 20 equidistant nodes allowed for robust fitting of individual MTA kinematics. The number of nodes has to be chosen considering the number of spline coefficients and the associated memory storage requirements, which vary exponentially with the number of GCs (i.e. $d$ ) and nodes (Table 7). However, during our tests, the total memory to store coefficients and nominal values for splines was smaller than that needed for $P^{e q}$. One spline per muscle is required to estimate both $\ell^{m t}$ and $r$ while $(d+1) P^{e q}$ are needed to individually fit $\ell^{m t}$ and $r$ nominal values (Menegaldo et al., 2004) (Table 7). This is an issue when nominal values are obtained from imaging techniques, with large storage requirements, and from which values for $r$ are difficult to determine.

As just suggested, one may use imaging methods to create splines from more realistic nominal values of $\ell^{m t}$. These could be determined using the centroids of three-dimensional muscle geometry created from MRI data (Blemker et al., 2007) or by three-dimensional geometric modeling such as host-mesh fitting methods (Oberhofer et al., 2010). Future developments may relax the limitation of splines needing equally spaced nodes, by using a generic spline basis as explained in (Habermann and Kindermann, 2007). 
Finally, our method is implemented in C++ with a MATLAB interface ${ }^{4}$ and is designed as a functional block that returns $\ell^{m t}$ and $r$ as a function of GC-angles facilitating the integration into large and complex musculoskeletal models.

\section{Supplementary Material}

Refer to Web version on PubMed Central for supplementary material.

\section{Acknowledgments}

The authors would like to thank Dr Elena Ceseracciu for her contribution to the implementation of the $\mathrm{C}++$ / MATLAB interface.

Funding sources included the US National Institute of Health (R01EB009351-01A2), the Australian National Health and Medical Research Council, the Western Australian Medical and Health Research Infrastructure Council, and the CARIPARO foundation in Italy.

\section{References}

Ackermann, M.; van den Bogert, AJ. Predictive simulation of gait in rehabilitation. Proceedings of the 32nd Annual International Conference of the IEEE Engineering in Medicine and Biology Society; Buenos Aires, Argentina. 2010.

Blemker SS, Asakawa DS, Gold GE, Delp SL. Image-based musculoskeletal modeling: applications, advances, and future opportunities. Journal of Magnetic Resonance Imaging. 2007; 25:441-451. [PubMed: 17260405]

Buchanan TS, Lloyd DG, Manal K, Besier TF. Neuromusculoskeletal modeling: estimation of muscle forces and joint moments and movements from measurements of neural command. Journal of Applied Biomechanics. 2004; 20:367-395. [PubMed: 16467928]

Delp SL, Anderson FC, Arnold AS, Loan P, Habib A, John CT, Guendelman E, Thelen DG. OpenSim: open-source software to create and analyze dynamic simulations of movement. IEEE Transactions on Biomedical Engineering. 2007; 54:1940-1950. [PubMed: 18018689]

Delp SL, Loan JP, Hoy MG, Zajac FE, Topp EL, Rosen JM. An interactive graphics-based model of the lower extremity to study orthopaedic surgical procedures. IEEE Transactions on Biomedical Engineering. 1990; 37:757-767. [PubMed: 2210784]

Fleischer C, Hommel G. A Human-Exoskeleton Interface Utilizing Electromyography. IEEE Transactions on Robotics. 2008; 24:827-882.

Gao F, Damsgaard M, Rasmussen J, Christensen ST. Computational method for muscle-path representation in musculoskeletal models. Biological cybernetics. 2002; 87:199-210. [PubMed: 12200615]

Garner BA, Pandy MG. The Obstacle-Set Method for Representing Muscle Paths in Musculoskeletal Models. Computer methods in biomechanics and biomedical engineering. 2000; 3:1-30. [PubMed: 11264836]

Habermann C, Kindermann F. Multidimensional Spline Interpolation: Theory and Applications. Computational Economics. 2007; 30:153-166.

Kuo AD. A least-squares estimation approach to improving the precision of inverse dynamics computations. Journal of Biomechanical Engineering-Transactions of the Asme. 1998; 120:148159.

Lloyd DG, Besier TF. An EMG-driven musculoskeletal model to estimate muscle forces and knee joint moments in vivo. Journal of Biomechanics. 2003; 36:765-776. [PubMed: 12742444]

McLean SG, Huang X, van den Bogert AJ. Association between lower extremity posture at contact and peak knee valgus moment during sidestepping: implications for ACL injury. Clinical Biomechanics. 2005; 20:863-870. [PubMed: 16005555]

\footnotetext{
${ }^{4}$ Spline and polynomial regression code, $\mathrm{C}++$ /MATAB interface, experimental data, and documentation are available as Supplementary Web Material from the Journal of Biomechanics web site.
} 
Menegaldo LL, de Toledo Fleury A, Weber HI. Moment arms and musculotendon lengths estimation for a three-dimensional lower-limb model. Journal of Biomechanics. 2004; 37:1447-1453. [PubMed: 15275854]

Menegaldo LL, de Toledo Fleury A, Weber HI. A 'cheap' optimal control approach to estimate muscle forces in musculoskeletal systems. Journal of Biomechanics. 2006; 39:1787-1795. [PubMed: 16033695]

Miller LH. Table of Percentage Points of Kolmogorov Statistics. Journal of the American Statistical Association. 1956; 51:111-121.

Murai A, Kurosaki K, Yamane K, Nakamura Y. Musculoskeletal-see-through mirror: computational modeling and algorithm for whole-body muscle activity visualization in real time. Progress in Biophysics and Molecular Biology. 2010; 103:310-317. [PubMed: 20869384]

Nordez A, Gallot T, Catheline S, Guevel A, Cornu C, Hug F. Electromechanical delay revisited using very high frame rate ultrasound. Journal of Applied Physiology. 2009; 106:1970-1975. [PubMed: 19359617]

Oberhofer K, Stott NS, Mithraratne K, Anderson IA. Subject-specific modelling of lower limb muscles in children with cerebral palsy. Clinical Biomechanics. 2010; 25:88-94. [PubMed: 19836868]

$\mathrm{S} \mathrm{H}$, Winter DA. A comparison of three muscle pennation assumptions and their effect on isometric and isotonic force. Journal of Biomechanics. 1991; 24:163-167.

Winby CR, Lloyd DG, Besier TF, Kirk TB. Muscle and external load contribution to knee joint contact loads during normal gait. Journal of Biomechanics. 2009; 42:2294-2300. [PubMed: 19647257]

Zajac FE. Muscle and tendon: properties, models, scaling, and application to biomechanics and motor control. Critical Reviews in Biomedical Engineering. 1989; 17:359-411. [PubMed: 2676342] 

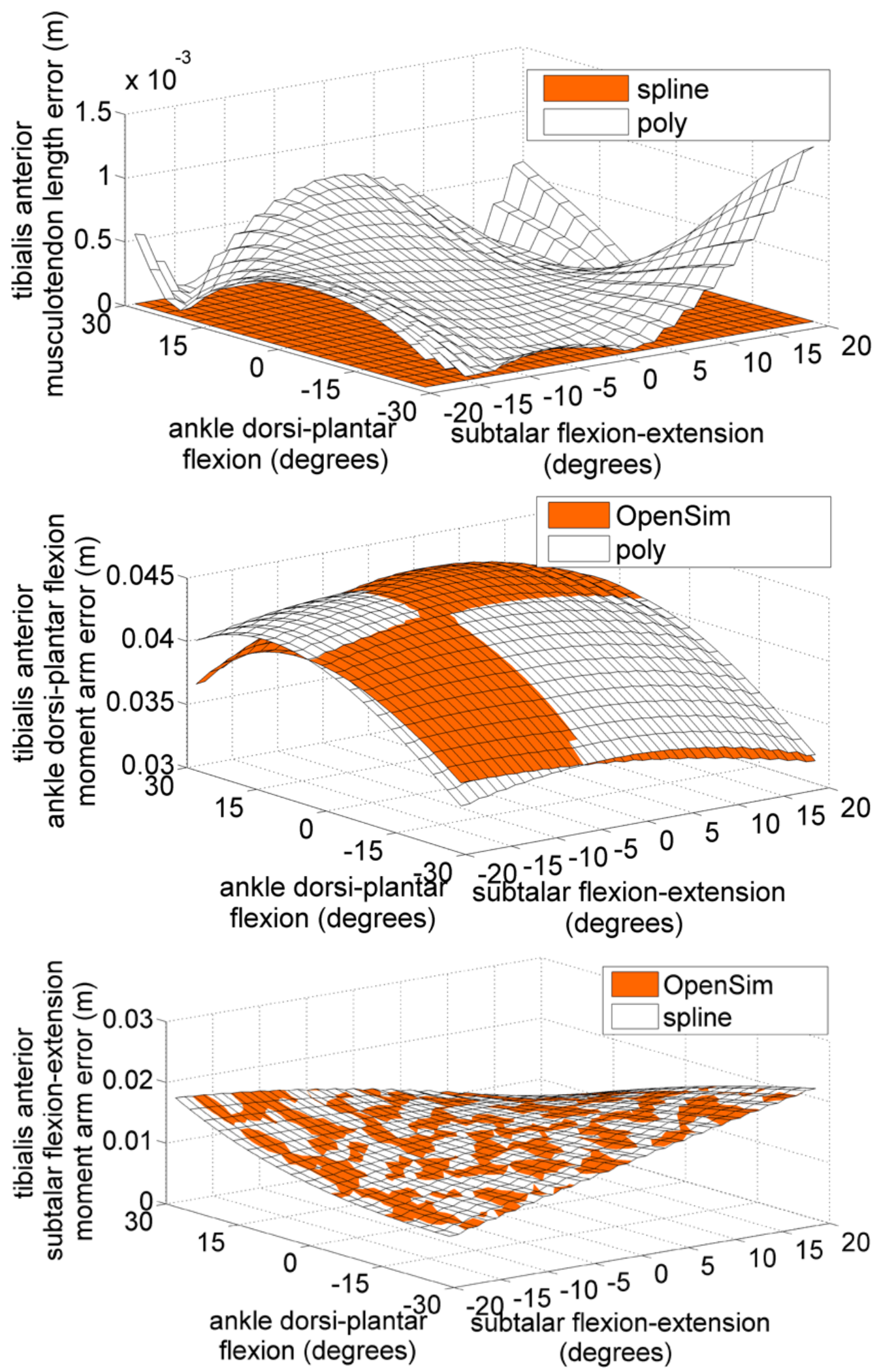

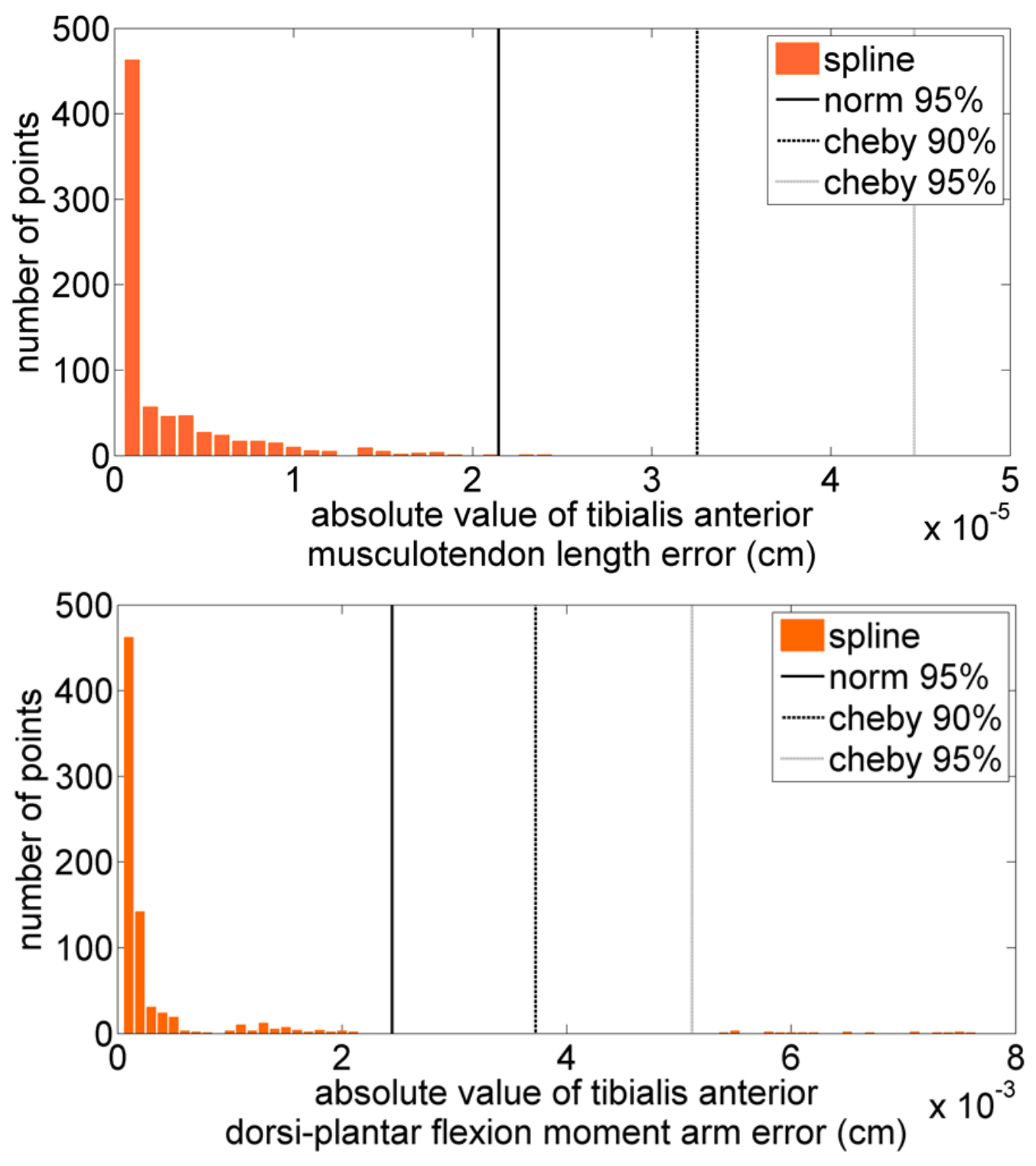


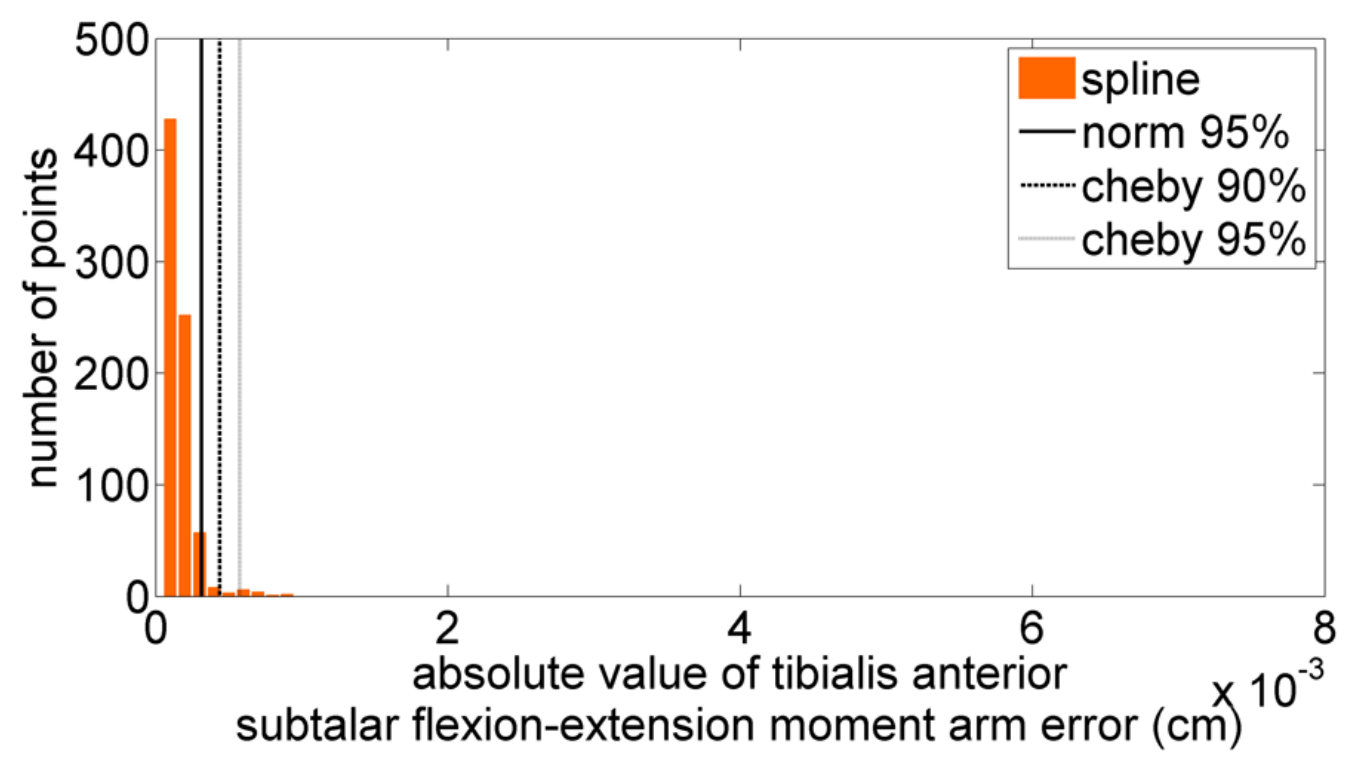

Figure 1.

First fitting accuracy test: all graphs report values for the tibialis anterior muscle using the LowerLimb4307 model (Delp et al., 1990). (a) The two surfaces represent the distribution of the difference between musculotendon length $\ell^{m t}$ computed using OpenSim and $\ell^{m t}$ estimated by $P^{e q}$ (poly) and spline function (spline) respectively. (b) The distribution of the ankle dorsi-plantar flexion moment arms $\left(r_{A F}\right)$ computed using OpenSim and using $P^{e q}$ respectively. (c) The distribution of the subtalar flexion-extension moment arm $\left(r_{S F}\right)$ computed using OpenSim and using the spline function respectively. Histograms (d), (e) and (f) show the spline method fitting error distribution associated to $\ell^{m t}, r_{A F}$ and $r_{\mathrm{SF}}$ respectively. The maximum expected errors (MEEs) are calculated both using Chebyshev's Theorem (cheby) and the Central Limit Theorem (norm). 

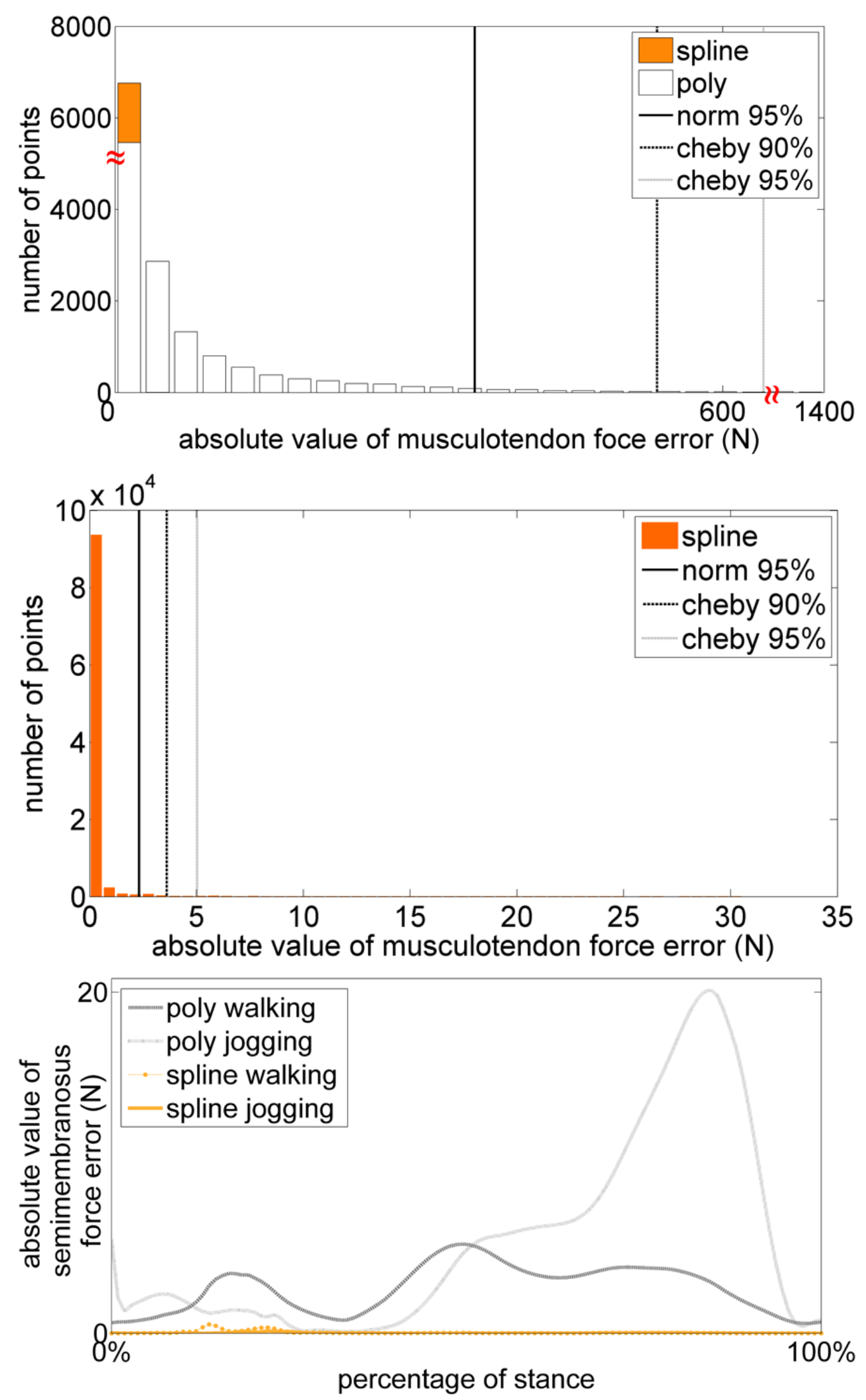

J Biomech. Author manuscript; available in PMC 2013 February 2. 
Figure 2.

Histograms (a) and (b) report the third test's error distribution associated to the prediction of the maximum isometric musculotendon force $F^{m t}$. The maximum expected error (MEE)

calculated both using Chebyshev's Theorem (cheby) and the Central Limit Theorem (norm) are also reported. Histogram (a) shows the error distribution for both spline and polynomial regression equations $P^{e q}$ (poly). MEEs are reported for $P^{e q}$ only because spline's MEEs are contained in the first (smallest) error class. Histogram (b) shows the error distribution for the spline method only as well as associated MEEs. Graph (c) reports the absolute value of the instantaneous semimembranosus force prediction errors averaged over all walking and jogging trials during the fourth test. Histograms (a) and (b), and graph (c) combine values for all biarticular thigh muscles in the LowerLimb1310 model (Table 5) (Winby et al., 2009). 


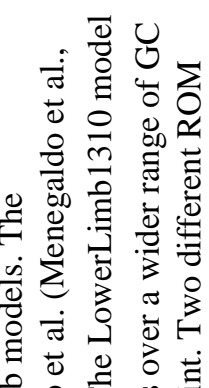

है ᄋ

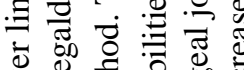

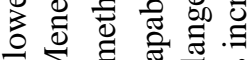

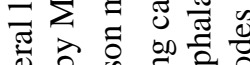

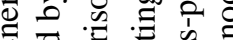

品

$\circ$ द्वे के बे

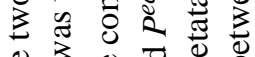

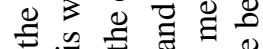

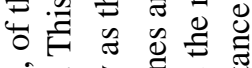

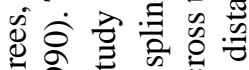

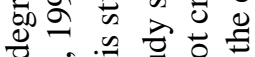

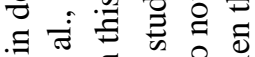

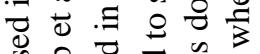

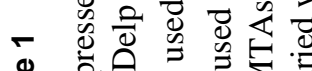

0

$0 \bigcup_{0}^{\infty} 30$

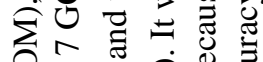

¿

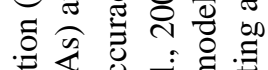

产芯宁完

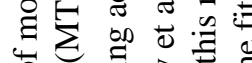

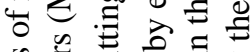

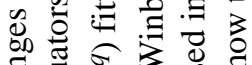

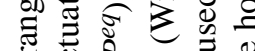

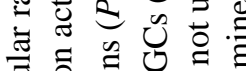

ప్

ส च

:

클 品

ธิษ

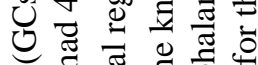

急

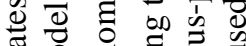

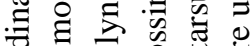

훙 矛

0 ० 0 एँ

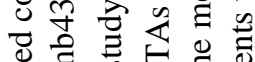

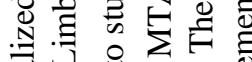

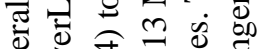

可方

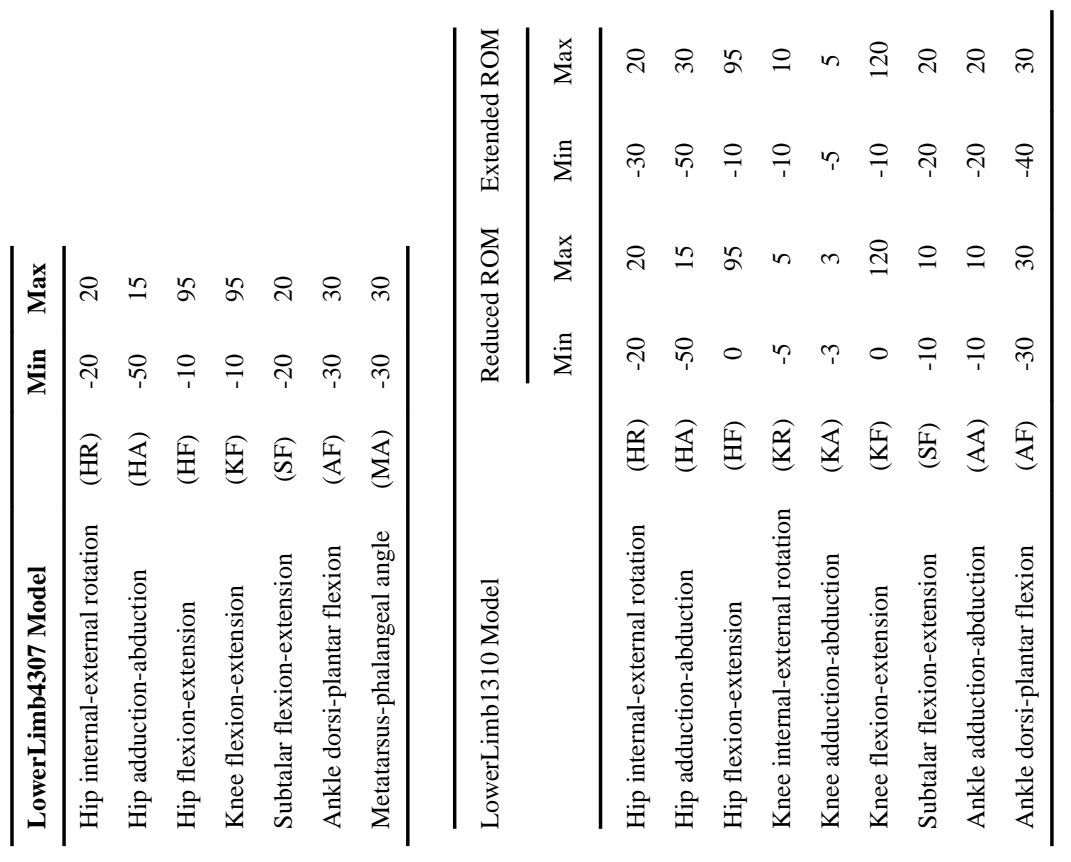




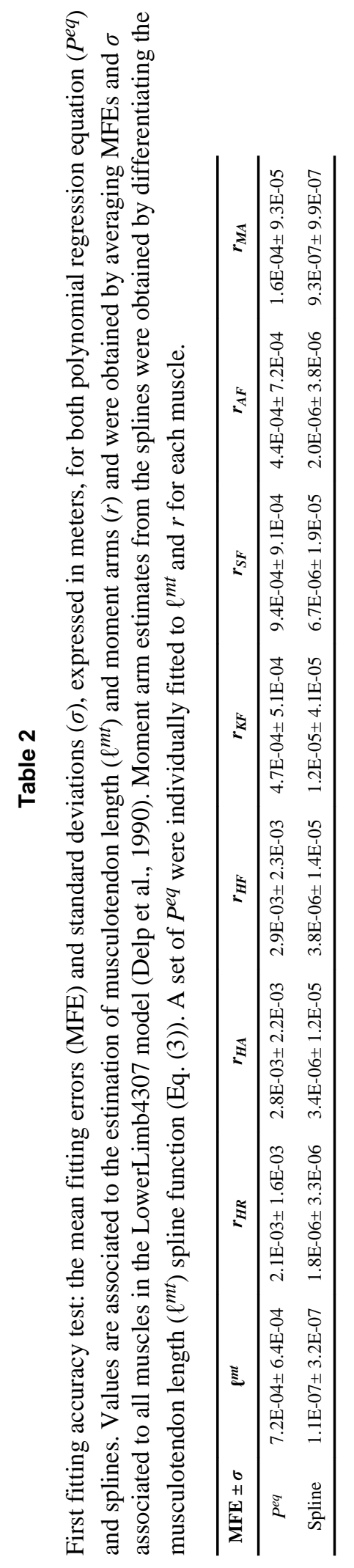

J Biomech. Author manuscript; available in PMC 2013 February 2. 

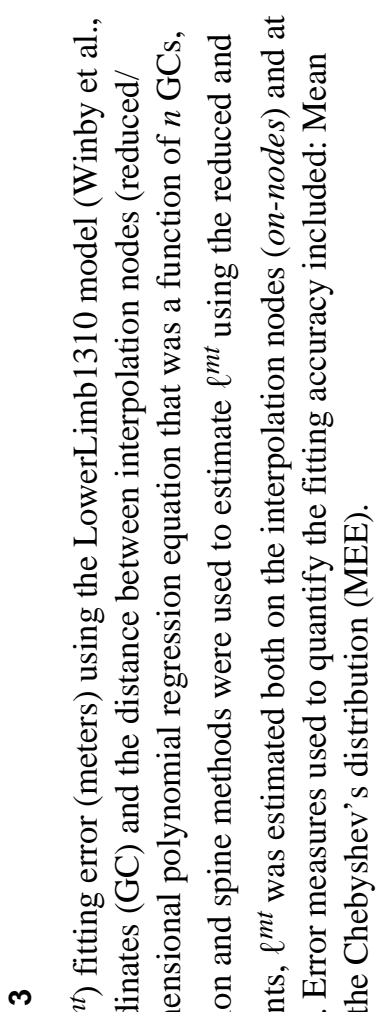

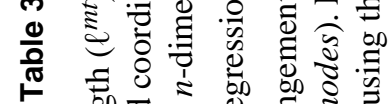

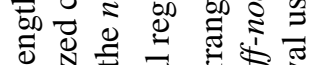

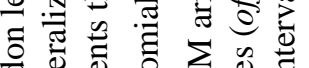
宅氙 ¿

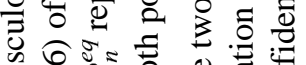

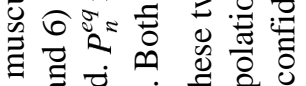

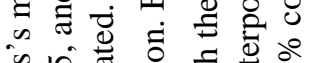

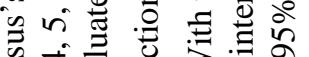

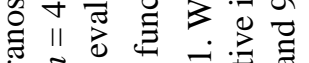
ป气⿻上丨 बี

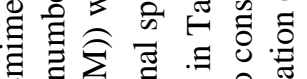
ప్ల

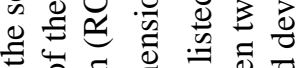
范苍苛矛

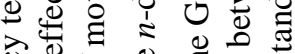

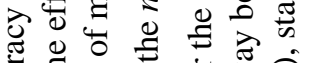

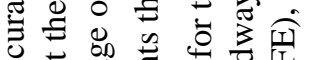

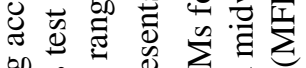

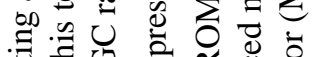

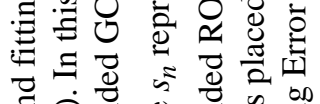
ठ웡

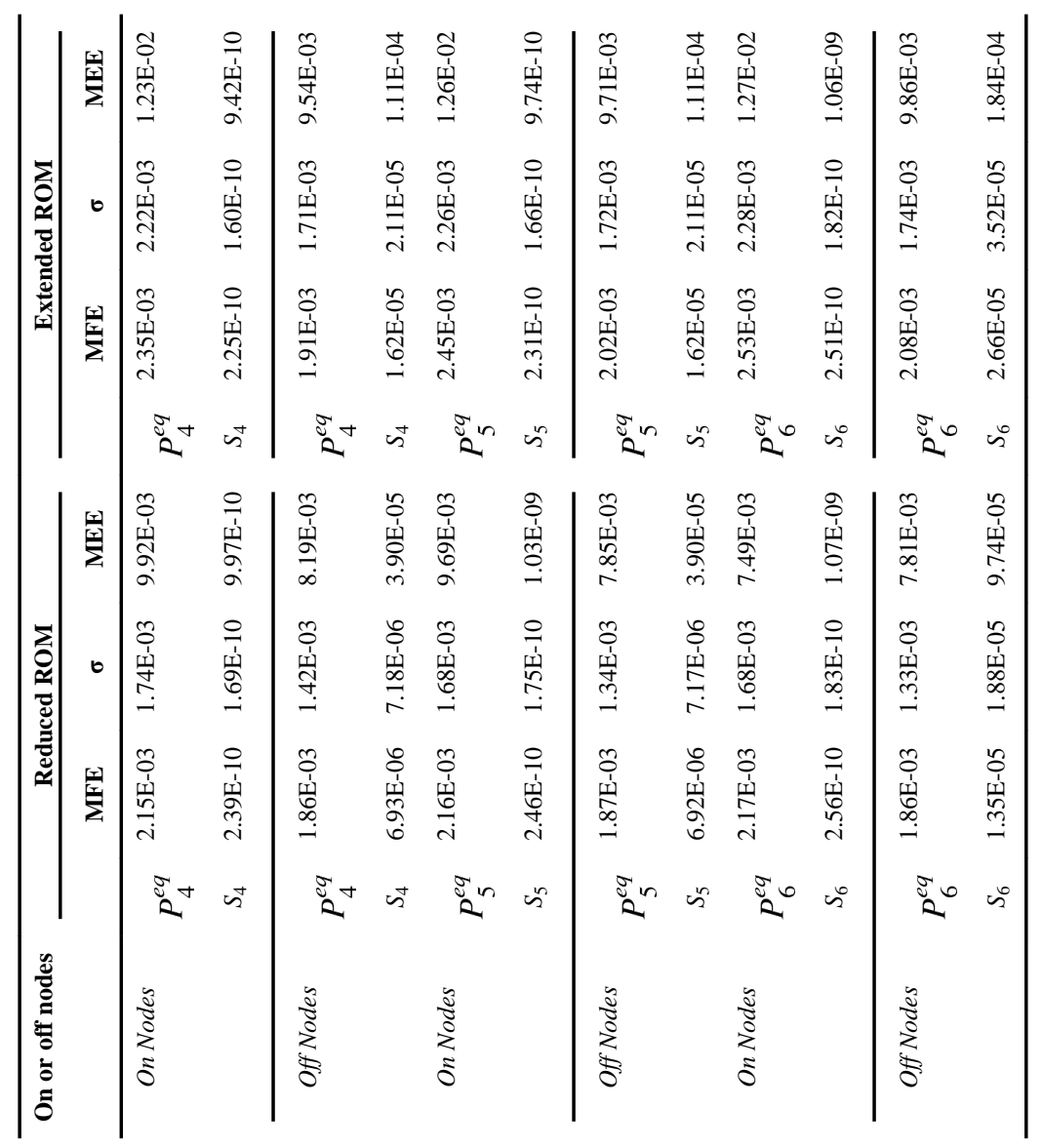




\section{Table 4}

Second fitting accuracy test: the percent mean fitting error $(\% \varepsilon)$ obtained using the LowerLimb1310 model (Winby et al., 2009). Values of $\% \varepsilon$ are from the estimation of the semimembranosus hip adduction-abduction moment arms $\left(r_{H A}\right)$. In this test the effect of the number $(n=4,5$, and 6$)$ of generalized coordinates $(\mathrm{GC})$ and the distance between interpolation nodes (reduced/extended GC range of motion (ROM)) were evaluated. Values of $\% \varepsilon$ are calculated using $P_{n}^{e q}$ that represents the $n$-dimensional polynomial regression equation that was a function of $n$ GCs and was used to directly estimate $r_{H A}$. Furthermore, values of $\% \varepsilon$ are also calculated using $\frac{\partial s_{n}^{l m t}}{\partial q_{H A}}$ that is the first-order partial derivative of the $n$-dimensional spline function that was used to directly estimate $\ell^{m t}$ (i.e. $s_{n}^{l m t}$ ) with respect to hip adduction-abduction GC $\left(q_{H A}\right)$. Values of $\% \varepsilon$ were calculated using both an extended and a reduced ROM as in Table 1. With these two arrangements, $\% \varepsilon$ was calculated both on the interpolation nodes (on-nodes) and on a set of nodes placed midway between the interpolation nodes (off-nodes).

\begin{tabular}{ccccc} 
& \multicolumn{2}{c}{ Reduced ROM } & \multicolumn{2}{c}{ Extended ROM } \\
\cline { 2 - 5 } & On-Nodes & Off-Nodes & On-Nodes & Off-Nodes \\
\hline$P_{4}^{e q}$ & 10.35 & 8.39 & 12.01 & 9.94 \\
$\frac{\partial s_{4}^{l m t}}{\partial q_{H A}}$ & 0.18 & 0.05 & 0.26 & 0.06 \\
\hline & & & & \\
$P_{5}^{e q}$ & 10.34 & 8.38 & 12.00 & 9.93 \\
$\frac{\partial s_{5}^{l m t}}{\partial q_{H A}}$ & 0.18 & 0.04 & 0.26 & 0.06 \\
\hline$\frac{P_{6}^{e q}}{\partial q_{H A}}$ & 10.34 & 8.38 & 12.00 & 9.95 \\
\hline$\frac{\partial s_{6}^{l m t}}{\partial q_{\text {HA }}}$ & 0.18 & 0.05 & 0.26 & 0.07 \\
\hline
\end{tabular}




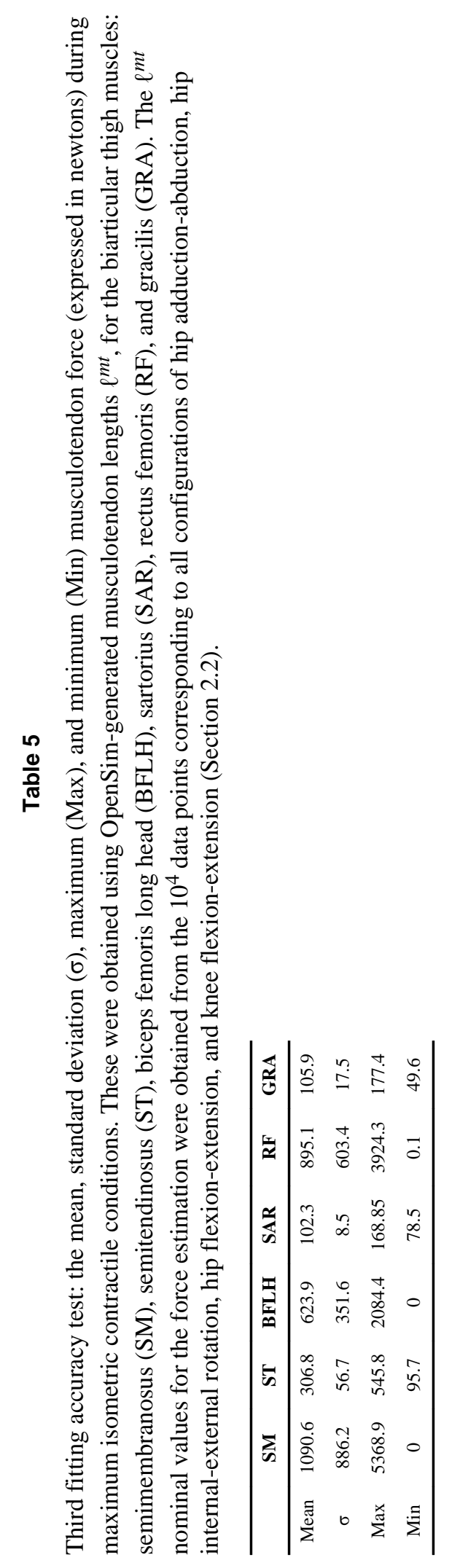

J Biomech. Author manuscript; available in PMC 2013 February 2. 


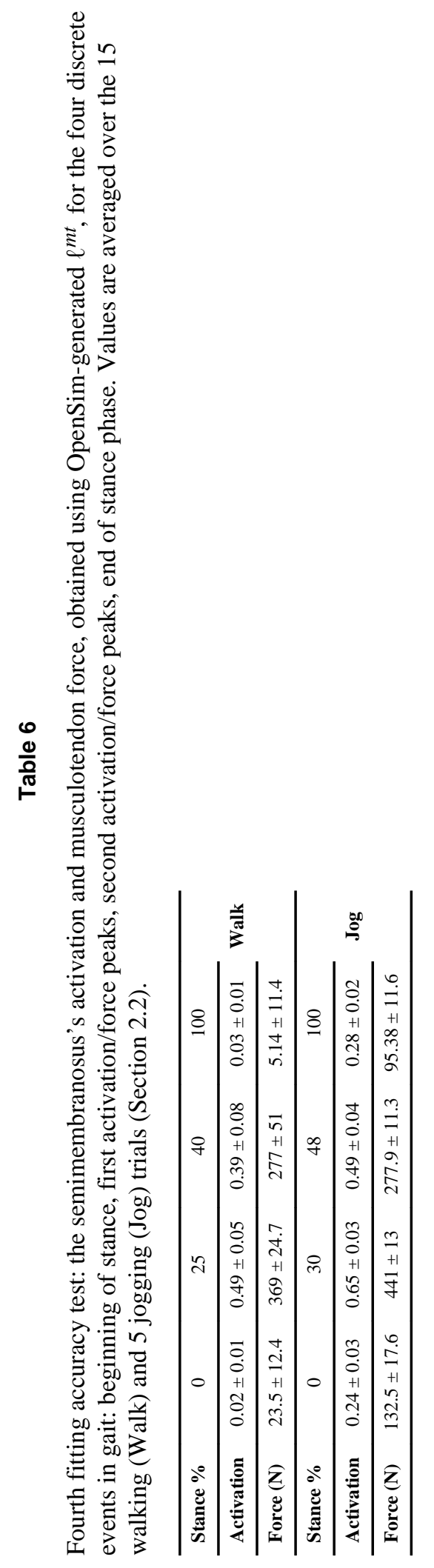

J Biomech. Author manuscript; available in PMC 2013 February 2. 


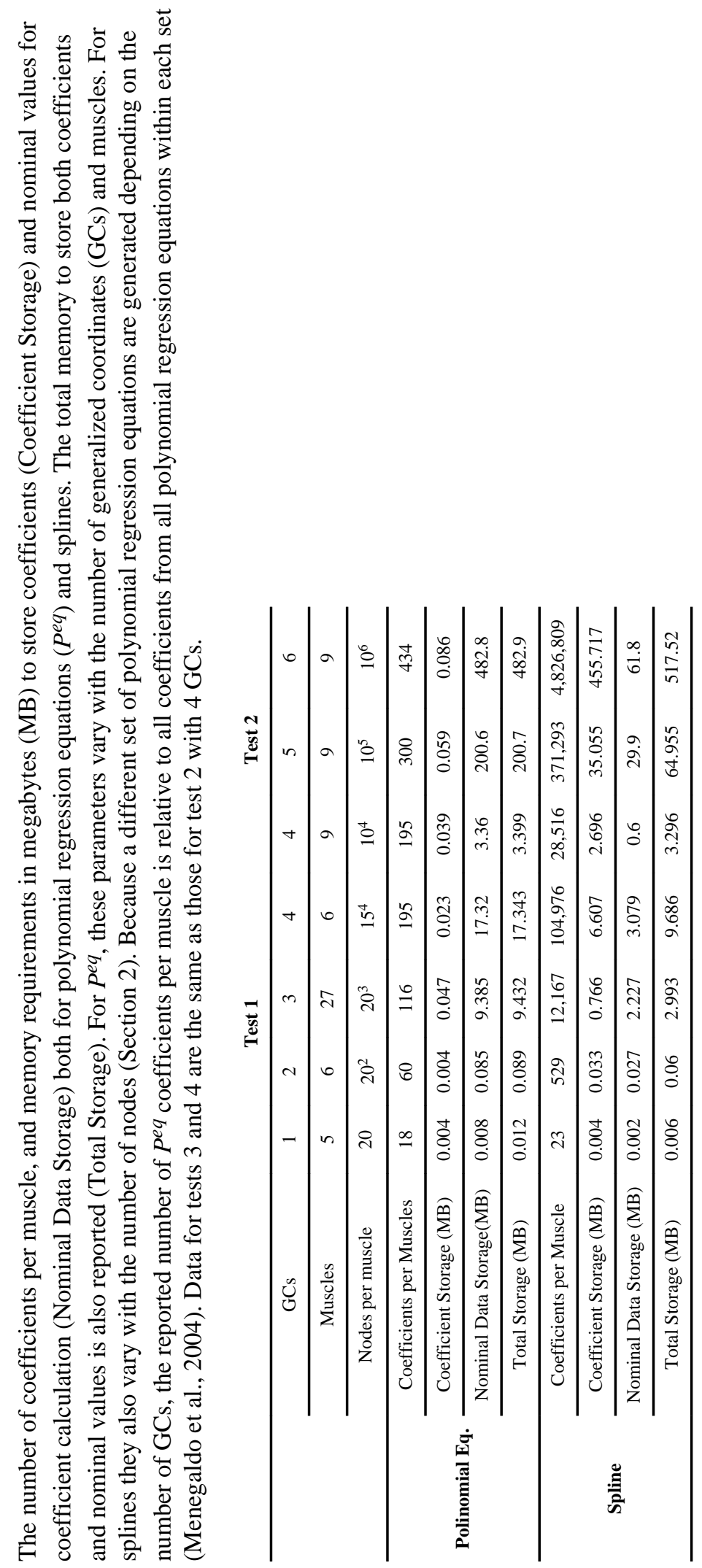

